\title{
What heavy quanta bounds could be inferred from a Higgs discovery?
}

\author{
J. Lorenzo Diaz-Cruz \\ Instituto de Fisica, BUAP, 72570 Puebla, Pue, Mexico
}

November 5, 2018

\begin{abstract}
The Higgs-gauge bosons couplings $g_{h V V}(V=g, \gamma, W, Z)$ receive non-decoupling corrections due to heavy quanta, and deviations from the SM predictions can be used to test its presence. The possible Higgs signal recently reported at LEP, with $m_{h}=115 \mathrm{GeV}$, severely constrains the presence of heavy quanta, such as a heavy fourth family. At Tevatron, the Higgs production by gluon fusion, followed by the decay $h \rightarrow W W^{*}$, can also be used to probe the existence of heavy colored particles, including additional standard or mirror families, chiral colored sextets and octet quarks. Within the MSSM, we also find that gluon fusion is a sensitive probe for the spectrum of squark masses.
\end{abstract}


1.- Introduction. The discovery of the Higgs boson as the remmant of the mechanism of electro-weak symmetry breaking (EWSB), is one of the most cherised goals of present and future high-energy experiments. Within the minimal standard model (SM), the mass of the physical Higgs particle is a free-parameter, but present indirect data seems to favor a moderate mass $\left(105<m_{h}<220 \mathrm{GeV}\right)$ 四, however this conclusion may be changed by the presence of new phyics with an scale $\Lambda \simeq O(1) \mathrm{TeV}$ [2]. On the other hand, the minimal supersymmetric version of the SM (MSSM), which is one of its most appealing extensions, predicts a light Higgs boson, with an upper mass bound of about $130 \mathrm{GeV}$ [3]. Detection of the full spectrum of Higgs bosons in the SM and beyond, constitute an important test of the possible realization of the Higgs mechanism in fundamental physics. In fact, a few events that could be interpreted as a SM Higgs signal were reported at the last stages of LEP 四.

The characteristic Higgs boson couplings determine the strategies employed for its search at present and future colliders. For instance, at LEP the reported Higgs events would come from the reaction $e^{+} e^{-} \rightarrow Z+h$, which takes advantage of the large couplings of the Higgs with massive gauge bosons. At Hadron colliders one can also test these couplings, either through the reactions $p p \rightarrow W+h, Z+h$, or through the decays $h \rightarrow W W, Z Z$; vector fusion (WW and ZZ) can also be used for heavy Higgs masses. The couplings of the Higgs with the heavier fermions, can be studied either by open production $(t \bar{t} h, b \bar{b} h)$ [5] or by the loop-induced Higgs coupling with gluon and photon pairs $(h g g, h \gamma \gamma)$ [6]. Any additional heavy particle that receives its mass from the SM Higgs mechanism, will couple to the Higgs with strength proportional to the particle mass itself, and will induce nondecoupling contributions to the 1-loop vertices $h g g, h \gamma \gamma$. These effects can play a significant role and may be probed at future colliders, as it has been explored in previous studies [8]. However, the presence of these new particles will also induce non-decoupling corrections to the tree vertices $h f \bar{f}, h W W$ and $h Z Z$ [9], which can affect the decay rate of detectable signatures, and thus must be included in the analysis. Beyond the SM, the lepton flavor violating Higgs decays $h \rightarrow l_{i} l_{j}$ could also be tested at colliders [7].

In this letter we are interested in studying the possible bounds on the presence of additional colored particles, that can be infered from the possible discovery of a Higgs signal, which then can become another tool for precision electro-weak studies. We consider first the possibility that the reported LEP 
Higgs signal is indeed true, and use it to constrain the presence of heavy quanta. Then, at hadron colliders we study the Higgs signal coming from the production by gluon fusion followed by the decay $h \rightarrow W W^{*}$, and find the bounds on heavy quanta that can be obtained from Tevatron (RUN-II). We also study gluon fusion for the MSSM higgs bosons, as a possible test for the mass spectrum of squarks; large effects are obtained when the loop amplitude includes non-degenerate squark masses, which could be realized in many particular models [10].

2.- Heavy quanta effects on Higgs vertices. In order to probe heavy scales through their effect on the Higgs coupling, we shall consider extensions of the SM that include additional particles, but with a minimal Higgs sector consisting of one doublet. The possible representations can only include fermion doublets and singlets [11], which can be arranged into: a) additional families with SM quantum numbers b) additional families with mirror properties, c) a combination of the above. Within cases a and b, new quarks must have the same color properties as in the SM, but within case c they could lay in larger $S U(3)_{c}$ representations, like sextets or octets.

Since these new states have electroweak charges, they will contribute in general to the parameters S,T,U, but not necessarily. Present global fits for electroweak data seem to exclude more than one additional SM family [12], however this conclusion relies on the assumption that no other physics occurs at the mass scale of the new fermions, which may not be the case in specific models, for instance in SUSY models. For illustration, we can consider an extended SUSY model with an additional (4th) family, and assume a mass degeneracy for both components of the fermion doublets, this gives $T=0$; whereas fermions give $S=2 N_{c} / 6 \pi$, the contribution of sfermions to $S$ is given by $S=\frac{N_{c}}{36 \pi} \log \left(\frac{m_{\tilde{u}_{L i}}^{2}}{m_{\tilde{d}_{L i}}^{2}}\right)[13$, thus by choosing the appropriate masses one could decrease the total value of $\mathrm{S}$, and satisfy present experimental constraints. Furthermore, including neutral majorana particles gives a negative contribution to S, and it makes even easier to satisfy present bounds. The light Higgs boson of the model $h^{0}$ behaves like the SM one in the limit $m_{A}>>m_{Z}$, under which the heavy Higgs bosons decouple.

Thus, we can identify two scenarios for our analysis, in Scenario-I we shall include a specific case of heavy quanta, namely a heavy fourth family, for which we can evaluate all the corrections induced on the vertices $h q \bar{q}$, $h g g / h \gamma \gamma$ and $h W W / h Z Z$, using the formalism of radiative corrections. The 
second case (Scenario-II) includes the situation when the mass of heavy colored particles comes from the SM Higgs boson, but which are weakly bounded by electroweak precision meassurements, i.e. we simply assume that there is some unspecified physics at the mass scale of the new particles that makes their contribution to the Peskin-Takeuchi parameters $S, T$ to be within experimental range [16].

For both scenarios, where we have additional heavy particles that receive their mass from the SM Higgs mechanism, but are weakly constrained by electroweak precision data, the study of the Higgs signal can test the presence of such heavy particles. To describe the effects of heavy quanta on the higgs couplings to gauge bosons, we shall write them in terms of the SM values $\left(g_{h V V}^{S M}\right)$ as: $g_{h V V}=g_{h V V}^{S M}\left(1+\epsilon_{V}\right)$, where $V=W, Z, g, \gamma$, and similarly for the fermion couplings $g_{h f f}=g_{h f f}^{S M}\left(1+\epsilon_{f}\right)$. The parameters $\epsilon_{f, V}$, encompass the effects of heavy quanta.

We could also consider effects arising from heavy particles that receive their mass from the breaking of new symmetries characterized by a large scale $V_{\text {new }}>>v=246 \mathrm{GeV}$, induced by another set of heavy Higgs bosons

$\left(\Phi_{\text {new }}\right)$. Supposse this is communicated to the SM Higgs by including in the scalar potential a mixing term of the form: $\lambda\left|\Phi_{n e w}\right|^{2}\left|\phi_{s m}\right|^{2}$. Then one can also include the contribution of these ultra-heavy particles to the parameters $\epsilon_{V}$. However, one can easily verify that in this case the mixing angle that relates the weak and mass-eigenstate Higgs basis, is suppressed by the large scale $\left(V_{\text {new }}\right)$, and it induces decoupling effects at low-energies, namely $\epsilon_{g} \simeq v / V_{\text {new }}$.

3.-Bounds on heavy quanta from the LEP Higgs signal. The possible discovery of a Higgs signal, with $m_{h}=115 \mathrm{geV}$, could be a remarkable closing for the series of sucessfull experiments performed at LEP. The reported events are indeed consistent with a SM higgs interpretation [幽, though a definite conclusion will have to wait for the coming stages of Tevatron RUN-II or even the LHC. Here, we shall assume that the Higgs signal is indeed real, and will obtain bounds on heavy quanta for Scenario-I. For the simple case of a heavy fourth family, one finds an interesting relation between $\epsilon_{f, W, Z}$ and the corresponding expression for the parameter $T$, namely,

$$
\begin{aligned}
\epsilon_{b} & =\frac{\alpha T}{2}+\frac{N_{c} G_{F}}{12 \sqrt{2} \pi^{2}}\left(m_{1}^{2}+m_{2}^{2}\right) \\
\epsilon_{Z} & =-\frac{N_{c} G_{F}}{6 \sqrt{2} \pi^{2}}\left(m_{1}^{2}+m_{2}^{2}\right)
\end{aligned}
$$




$$
\epsilon_{W}=\epsilon_{Z}+\frac{\alpha T}{2}
$$

where $T$ includes the contribution from the fourth family fermions, with masses $m_{1,2}$. It is interesting to comment that this relation holds for any other colored heavy family that transform as doublets and singlets under $S U(2)_{L} \times U(1)_{Y}$.

Then, to evaluate the event rate for Higgs production at LEP, we find convenient to write the product of the cross-section for $e^{+} e^{-} \rightarrow Z H$ times the b.r. of $h \rightarrow b b$, in terms of the SM result, namely: $[\sigma \times B . R .(h \rightarrow$ $b b)]_{\text {new }}=R_{Z b} \times[\sigma \times B . R .(h \rightarrow b b)]_{S M}$, where $R_{Z b}$ is given by

$$
R_{Z b}=\frac{\left(1+\epsilon_{Z}\right)^{2}\left(1+\epsilon_{b}\right)^{2}}{\left[1+\sum_{Y}\left(\epsilon_{Y}^{2}+2 \epsilon_{Y}\right) * B \cdot R \cdot\left(h_{S M} \rightarrow Y Y\right)\right]},
$$

the sum in the denominator runs over the allowed modes $(Y=t, b, g, A, W, Z)$. Results for $R_{Z b}$ corresponding to a heavy fourth quark generation, and $m_{h}=$ $115 \mathrm{GeV}$, are shown in Table 1 . Then, by comparing the event rate reported at LEP, which seems consistent with the SM Higgs (for which $R_{Z b}=1$ ), and the value for $R_{Z b}$ predicted in the presence of the heavy quanta, one could test their presence. From Table 1, we can see that by just having a $25 \%$ precision on the cross-section determined by LEP, it would be possible to exclude a heavy fourth generation, with masses above $150 \mathrm{GeV}$, which is a remarkable result.

4.- Bounds on heavy quanta at Tevatron. At hadron colliders we are interested in evaluating the effects of heavy quanta on the Higgs production by gluon fusion. In general, the expression for the correction to the couplings $h g g\left(\epsilon_{g}\right)$ is given by a ratio of complicated loop expresions, however since we are interested in the limit $m_{h}<<M_{\text {heavy, }}$, one can use the low-energy theorems [14, to relate $\epsilon_{g}$ to the beta-function coefficients for the strong coupling constant $\left(\beta_{3, X}\right)$. Then, we have $\epsilon_{g}=\beta_{3, X} / \beta_{3, t}$, where $\beta_{3, t(X)}$ denote the contribution of top and heavy quanta $(\mathrm{X})$ to the strong beta function. The values of $\epsilon_{g}$ arising from a pair of heavy color triplets, sextets and octet quarks are : $\epsilon_{g}=2,5$ and 6, respectively, whereas a new SM family plus its mirror partner gives $\epsilon_{g}=4$. Thus, the new particles will modify the cross section by gluon fusion, which can be written in terms of the SM result $\left(\sigma_{S M}\right.$ ) as: $\sigma(p p \rightarrow h+X)=\sigma_{S M}\left(1+\epsilon_{g}\right)^{2}$. New colored particles give $\epsilon_{g}>0$ and will enhance the corresponding cross-section. 
Bounds on the effects of heavy quanta can be obtained at Tevatron using gluon fusion and the decay $h \rightarrow W W^{*}$, which was studied in detail for the SM in ref. [17], this work concluded that it is possible to detect a SM Higgs boson with an integrated luminosity of $30 \mathrm{fb}^{-1}$, provided that an optimized selection of cuts is implemented; here we shall only use their first stage of cuts, namely: transverse lepton momentum $p_{t}(e, \mu)>10 \mathrm{GeV}$, psedorapidity $\eta_{e, \mu}<1.5$, lepton invariant mass $m_{l l}>10 \mathrm{GeV}$, jet resolution $\Delta R(l-j)>0.4$, missing transverse energy $E_{T}>10 \mathrm{GeV}$.

For Scenario-I (the fourth family case), the corrections to the vertex $h W W$ are negative (as compared with the positive tree-level value) and grow with the mass of the heavy quanta; for instance for fermion masses of order 500 (700) GeV the deviations from the SM tree-level couplings are of order - 27 (-52) \%, which seems to imply that the signal from $h \rightarrow W W^{*}$ will no longer be detectable, i.e. one can only probe heavy but not ultraheavy quanta. With $10 \mathrm{fb}^{-1}$ of integrated luminosity, it will be possible to exclude only a certain range of fermion masses, up to about $900 \mathrm{GeV}$, as it is shown in table 1 . On the other hand, the radiative corrections to the vertex $h b \bar{b}$ show the opposite behaviour, namely they grow with the heavy fermion masses; however, it will be difficult to detect this effect at hadron colliders for the intermediate Higgs masses considered here, since B.R. $(h \rightarrow b \bar{b}) \simeq 1$. Rather than focusing on the B.R., one has to look for meassuring the Higgs width, which only seems possible at a muon-collider, where the Higgs would be produced as an s-channel resonance, which is directly sensitive to $\Gamma(h \rightarrow b \bar{b})$.

On the other hand, for the case when the corrections to $\epsilon_{W}$ can be neglected (Scenario-II), the above cuts already allow us to get interesting bounds on the parameter $\epsilon_{g}$ at $95 \%$ c.l., as it is shown in figure 1 ( for 2 and $\left.10 \mathrm{fb}^{-1}\right)$. These bounds will in turn constrain the presence of heavy colored particles, provided that the Higgs mass lays in the intermediate mass range. The interception of the exclusion lines with the straight dashed line (which corresponds to $\epsilon_{g}=2$ ), shows the values of Higgs masses for which it will be possible to limit the presence of a pair of heavy color triplets. Furthermore, since sextets and octet quarks give a larger value of $\epsilon_{g}$, their presence could be excluded too. It is remarkable that coming stages of Tevatron will be able to test $\epsilon_{g}$ at significant levels.

5.- Probing a Non-universal sfermion spectrum. The MSSM includes two Higgs doublets, and the Higgs spectrum consists of two neutral CP-even scalars $h^{0}$ and $H^{0}$, one CP-odd pseudoscalar $A^{0}$ and a charged pair 
$H^{ \pm}$[18]. The Higgs sector of the model is completely determined at tree level by fixing two parameters, conventionally chosen to be $\tan \beta$ and the pseudoscalar mass $m_{A}$. At loop levels, the radiative corrections, mainly from top and stop, modify the tree-level mass bound $\left(m_{h}<m_{Z} \cos 2 \beta\right)$, allowing to have $m_{h}^{\max } \simeq 130 \mathrm{GeV}[3]$. The corrections to Higgs couplings can also lead to modification of its production mechanisms at hadron colliders, as previously studied [19]. Although conventional wisdom states that the contribution of top/bottom quarks (squarks) dominate the amplitude for the gluon fusion, and squarks from first and second generations can be neglected, it should be mentioned that this result only holds within the minimal SUSY breaking scenarios, where it is typically assumed that sfermions are mass degenerate, as required to respect FCNC constraints [20]. However, mass non-degeneracy for sfermions within the same family but different isospin can be acceptable, since it will only be midly constrained by the parameter $T$. In fact, some non-degeneracy can arises even in the minimal SUSY-GUT, by imposing universal boundary conditions at the Planck scale, rather than the GUT scale [10].

In this letter we consider the decay $h \rightarrow g g$ (which determines the gluon fusion mechanism) to probe the structure of soft-breaking masses predicted by models of SUSY breaking. The expression for the decay width of $h \rightarrow g g$ that includes a non-universal mass spectrum for squarks, can be written as,

$$
\Gamma(h \rightarrow g g)=\frac{G_{F} \alpha_{s}^{2} m_{h}^{3}}{4 \sqrt{2} \pi}\left|G_{h}\right|^{2}
$$

where

$$
G_{h^{0}}=G_{t, \tilde{t}}+G_{b, \tilde{b}}+G_{L R}+G_{U D}
$$

For $h=h^{0}, G_{h}$ is given by:

$$
\begin{aligned}
G_{t, \tilde{t}}= & -\frac{m_{t}^{2}}{m_{h}^{2}} \frac{\cos \alpha}{\sin \beta}\left[f_{1}\left(\lambda_{t}\right)+f_{3}\left(\lambda_{\tilde{t}_{L}}\right)+f_{3}\left(\lambda_{\tilde{t}_{R}}\right)\right] \\
G_{b, \tilde{b}}= & \frac{m_{b}^{2}}{m_{h}^{2}} \frac{\sin \alpha}{\cos \beta}\left[f_{1}\left(\lambda_{b}\right)+f_{3}\left(\lambda_{\tilde{b}_{L}}\right)+f_{3}\left(\lambda_{\tilde{b}_{R}}\right)\right] \\
G_{L R}= & \frac{m_{W}^{2} s_{w}^{2}}{m_{h}^{2} c_{W}^{2}} \sin (\beta+\alpha)\left[Q _ { u } \left(f_{3}\left(\lambda_{\tilde{u}_{R}}\right)-f_{3}\left(\lambda_{\tilde{u}_{L}}\right)\right.\right. \\
& +Q_{d}\left(f_{3}\left(\lambda_{\tilde{d}_{R}}\right)-f_{3}\left(\lambda_{\tilde{d}_{L}}\right)\right]
\end{aligned}
$$




$$
G_{U D}=\frac{m_{W}^{2}}{2 m_{h}^{2} c_{W}^{2}} \sin (\beta+\alpha) \Sigma_{u, d}\left[f_{3}\left(\lambda_{\tilde{u}_{L}}\right)-f_{3}\left(\lambda_{\tilde{d}_{L}}\right)\right]
$$

The expression for $h=H^{0}$ are obtained by making the replacements $\cos \alpha \rightarrow$ $-\sin \alpha, \sin \alpha \rightarrow-\cos \alpha, \sin (\alpha+\beta) \rightarrow-\cos (\alpha+\beta)$; the explicit form of $f_{1}(z), f_{3}(z)$ can be found in ref. [15]. The contribution proportional to the fermion masses are kept only for the stop and sbottom $\left(G_{t, \tilde{t}}, G_{b, \tilde{b}}\right)$, whereas the remmaining SUSY-breaking effects are kept for all sfermions $\left(G_{L R}, G_{U D}\right)$. To reduce the number of parameters, we choose our $\mu, A_{t}$ values in such a way that one can neglect L-R mixing, which was found previously to give small effects [19]. We have used the previous equation to evaluate the contribution of squarks to the parameter $\epsilon_{g}$, for $m_{A}=200 \mathrm{GeV}$, $\tan \beta=5,20$, and squark masses covering the range from 200 to $700 \mathrm{GeV}$. For simplicity, we apply the 1-loop leading log formula to the Higgs masses and parameters. Results are shown in table 2, and for comparision we have also included the values obtained with universal squark masses. These results show that the effect of non-universal masses can modify the B.R. $(h \rightarrow g g)$, by values of order $\pm 20 \%$.

6.- Conclusions. We have studied the Higgs interaction with fermions and gauge bosons, and found that deviations from the SM prediction, induced by heavy quanta, can be described by a set of parameters $\epsilon_{f, V}$, which can be tested at future colliders. The Higgs signal reported at LEP can exclude a heavy fourth family, wheres Tevatron can also be used to probe heavy quanta through the Higgs production by gluon fusion, followed by the promising Higgs decays into $W W^{*}$. The resulting bounds can imply the exclusion of heavy particles that receive their mass directly from the SM Higgs, including a 4th standard or mirror family or chiral colored sextets and octets. For the 4th family case we disply a relations between $\epsilon_{f, V}$ (the parameters that describe the corrections to the Higgs coupling) and the Peskin-Takeuchi parameter $T$. Within the MSSM, we also find that gluon fusion is a sensitive probe for a non-universal spectrum of squarks masses, which allows for the possibility to test the sfermion masses predicted in the models of SUSY breaking.

We thank M. Chanowitz and C. Kolda for useful discussions. Work supported by CONACYT-SNI (Mexico). 


\section{References}

[1] For a recent review see: M. Kado, talk at Recontres de Moriond (March, 2000), hep-ex/0005022.

[2] J.L Diaz-Cruz et al., Mod. Phys. Lett. A15 (2000) 1377 hep-ph/9905335; C. Kolda and L. Hall, Phys. Lett. B459 (1999) 213 hep-ph/9904236.

[3] For an updated calculation and references to earlier work see: M. Carena et al., hep-ph/0001002; see also: J.R. Espinosa and Ren-Jie Zhang, Nucl. Phys. B586 (2000) 3.

[4] See for instance the talk by P. Igo-Kemenes, "Statuts of the Higgs boson searches" (ALEPH, DELPHI, L3, OPAL and the LEP Higgs working group), Nov. 3, 2000.

[5] J.L. Diaz-Cruz et al., Phys. Rev. Lett. 80 (1998) 4641; C. Balaaz et al., Phys. Rev. D59 (1999) 055016; M. Carena, S. Mrenna and C. Wagner, Phys. Rev. D60 (1999) 075010; see also: M Carena et al, "Report of the Tevatron Higgs working group", hep-ph/0010338.

[6] J. Gunion, H. Haber, G. Kane and S. Dawson, 'The Higgs Hunters Guide', Adison Wesley, Reading 1990.

[7] J.L. Diaz-Cruz and J. Toscano, Phys. Rev. D62 (2000) 116005 ; M. Sher, Phys. Lett. B487 (2000) 151; T. Han and D. Marfatia, Phys. Rev. Lett. 86 (2001) 1442.

[8] M. Chanowitz, Phys. Rev. Lett. 69 (1992) 2037; I.F. Ginzburg, I.P. Ivanov and A. Shiller, Phys. Rev. D60 (1999) 095001; S. Dawson and H. Haber, Phys. Rev. D44 (1991) 53.

[9] M. Veltman, Nucl. Phys. B123 (1977) 89; M. Chanowitz, M. Furman and I. Hinchliffe, Nucl. Phys. B153 (1979) 402.

[10] N. Polonski and A. Pomarol, Phys. Rev. D51 (1995) 6532.

[11] J. A. Minahan, P. Ramond and B.D. Wright, Phys. Rev. D42 (1990) 1692. 
[12] J. Erler and P. Langacker, in Review of Particle Properties, Eur. Phys. J C3 (1998) 1.

[13] A. Dobado, M.J. Herrero and S. Penaranda, Eur. Phys. J. C7 (1999) 313.

[14] A. Vainshtein et al. Sov. J. Nucl. Phys. 30 (1979) 711; B. Kniehl and M. Spira Z. Phys. C69 (1995) 77.

[15] For a review see: M. Spira, Fortsch. Phys. 46 (1998) 203.

[16] M. Peskin and T. Takeuchi, Phys. Rev. Lett. 65 (1990) 964.

[17] T. Han, A. Turcot and R.J. Zhang, hep-ph/9812275.

[18] J. Gunion and H. Haber, Nucl. Phys. B272 (1986) 1.

[19] A. Djouadi, Phys. Lett. B435 (1998) 101.

[20] A. Masiero et al., Nucl. Phys. B477 (1996) 321.

\section{FIGURE CAPTION}

Fig 1. $95 \%$ exclusion contours for the parameter $\epsilon_{g}$ that can be obtained from Higgs search at Tevatron RUN-II for Scenario-II, with 2 (dashes) and 10 (solid) $\mathrm{fb}^{-1}$ of integrated luminosity. The straight (dashed) lines corresponds to $\epsilon_{g}=2$.

\section{TABLE CAPTION}

Table 1. Values of $R_{Z b}$ obtained for a 4th generation of quarks, for a Higgs mass $m_{h}=115 \mathrm{GeV}$ (Scenario-I).

Table 2. Upper limits to 4th generation fermion masses that can be obtained from Higgs search at Tevatron RUN-II, with $10 \mathrm{fb}^{-1}$ (Scenario-I).

Table 3. Values predicted for the parameter $\epsilon_{g}$ for the MSSM with $\tan \beta=$ 5, 20. $M_{\tilde{Q}}$ represents the range of values taken for $M_{\tilde{U}_{R}}, M_{\tilde{D}_{L}}, M_{\tilde{D}_{R}}$.

Table. 1 


\begin{tabular}{||l|l||}
\hline$m_{4 t h}[\mathrm{GeV}]$ & $R_{Z b}$ \\
\hline 150. & 0.74 \\
\hline 200. & 0.72 \\
\hline 250. & 0.70 \\
\hline 300. & 0.67 \\
\hline 350. & 0.64 \\
\hline 400. & 0.60 \\
\hline
\end{tabular}

Table. 2

\begin{tabular}{||l|l||}
\hline$m_{h}[\mathrm{GeV}]$ & $m_{4 t h}^{\max }[\mathrm{GeV}]$ \\
\hline 120. & 350. \\
\hline 130. & 520. \\
\hline 140. & 650. \\
\hline 150. & 730. \\
\hline 160. & 870. \\
\hline 170. & 710. \\
\hline 180. & 670. \\
\hline 190. & 600. \\
\hline 200. & 510. \\
\hline
\end{tabular}

Table. 3

\begin{tabular}{||l|l|c|l||}
\hline$m_{\tilde{U}}[\mathrm{GeV}]$ & $m_{\tilde{Q}}[\mathrm{GeV}]$ & $\epsilon_{g}$ (non-univ) & $\epsilon_{g}$ (univ) \\
\hline 200. & $200 \rightarrow 700$ & $0.1 \rightarrow 0.4$ & 0.4 \\
\hline 500. & $200 \rightarrow 700$ & $-0.05 \rightarrow 0.14$ & 0.05 \\
\hline 700. & $200 \rightarrow 700$ & $-0.05 \rightarrow 0.18$ & 0.1 \\
\hline
\end{tabular}




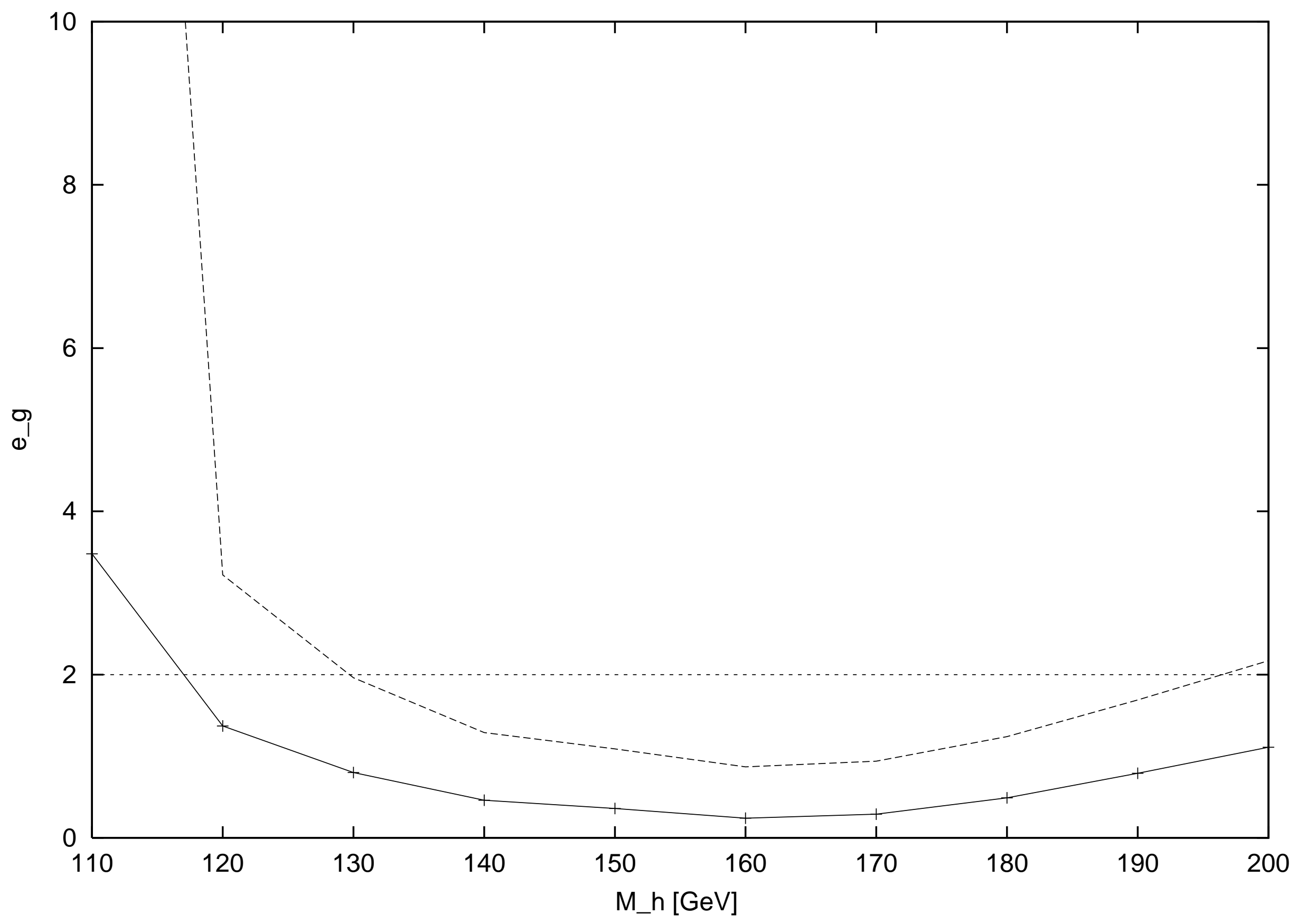

